\title{
Motion-blurred star image acquisition and restoration method based on the separable kernel
}

\author{
Honglin Yuan ${ }^{\mathrm{a}}$, Fan $\mathrm{Li}^{\mathrm{b}}$ and Tao $\mathrm{Yu}^{\mathrm{c}}$ \\ Beihang University, Beijing 100191, China. \\ ayuanhonglin@buaa.edu.cn, ${ }^{b}$ lifan@buaa.edu.cn, cytrock@buaa.edu.cn
}

Keywords: star tracker, non-uniform motion blur, simulation, restoration.

\begin{abstract}
The star tracker is supposed to have the highest accuracy among different types of attitude measurement devices. However, images taken by the star tracker under dynamic conditions are often blurred, especially in deep space exploration, which limits its high dynamic performance and accuracy. This paper addresses how to simulate blurred star images and remove image blurs that arise when the star tracker is under dynamic conditions of angular vibrations and rotations. We classify and model star tracker's rotation and angular vibration. After that, we simulate the blurred star images caused by composite motion of rotations and angular vibrations. After acquiring the motion-blurred star image, a restoration method is proposed to restore it. The simulation results indicate that our approach makes it possible to model and remove a wider class of blurs than previous approaches, including uniform blur as a special case and the extraction accuracy of star spot centroid is improved significantly using the restored star image compared with the blurred one.
\end{abstract}

\section{Introduction}

Attitude determination plays an important role on the optical autonomous navigation, particularly in urgent demand for deep-space mission. The star tracker is supposed to have the highest accuracy under stable conditions among different types of attitude measurement devices [1]. Tradition approaches for star centroid extraction require enough energy and good shape of stars. However, in deep-space exploration, the apparent magnitude of navigation stars used for optical autonomous navigation is high, the exposure time and focal length are long, these limiting star trackers' update rate and dynamic performance [2], which is more likely to lead to blurred star images. However, it is not helpful to use traditional mechanical, optical approaches [3, 4] which are based on hardware to restore the motion-blurred star image. For these reasons, studying the motion-blurred star image acquisition and restoration methods have important theoretical meaning and engineering application value for the star tracker and is helpful to improve the star tracker' dynamic performance.

While significant progress has been made recently towards simulating motion-blurred star images, most of current approaches model the blurred star image as the convolution of a sharp image with a spatially uniform kernel. Zhang [5] simulated spatially varying motion-blurred star images, but only the rotation was considered. Sun [6] proposed a smearing motion model of the star spot and only simulated blurred star images with uniform blur kernel. Wang [7] built motion-blurred models and then simulated multiple blurred star images which contained both uniform and non-uniform blur kernels. However, his method cannot exactly describe the star tracker's motion path.

If the blur kernel for each pixel has the same form, the problem of restoration is generally referred to as no-blind deconvolution, for which standard techniques such as the Wiener filter[8] or the Richardson-Lucy[9] exists. However, these restoration algorithms have generally been limited to the case of uniform blur. To restore images with spatial-variant blurs, Wang [7] used the step-by-step method to deblur the motion-blurred star images, but this method is not useful when the blurred images contain non-uniform blurs. 
All in all, recent work mostly focuses on simulation and restoration of blurred star images containing uniform blur caused by single angular motion or vibration. However, little research has been done on blurred star image caused by the combine of rotation and angular vibration.

\section{Simulation method of the blurred star image}

In this section, we use an integration of the clear star image under a sequence of planar projective transforms to get the blurred star image.

Supposing that an image $f(x, y)$ undergoes planar motion and $x_{o}(\mathrm{t})$ and $y_{o}(\mathrm{t})$ are the time-varying components of motion in the $\mathrm{X}$ and $\mathrm{Y}$ directions respectively, the total exposure at any point of the CCD is obtained by integrating the instantaneous exposure over the time interval during which the image system shutter is open. $T$ is the exposure time and the blurred star image $g(x, y)$ can be obtained by[10]

$$
g(x, y)=\int_{0}^{T} f\left[x-x_{0}(t), y-y_{0}(t)\right] d t
$$

Subdividing exposure time $T$ into $M$ equal portions, it follows that

$$
\begin{aligned}
g(x, y) & =\int_{0}^{T} f\left[x-x_{0}(t), y-y_{0}(t)\right] d t \\
& =\frac{1}{M} \sum_{i=0}^{M-1} f\left[x-x_{0}\left(t_{i}\right), y-y_{0}\left(t_{i}\right)\right]
\end{aligned}
$$

where $t_{M-1}=T$ and $f\left[x-x_{0}\left(t_{i}\right), y-y_{0}\left(t_{i}\right)\right]$ is the projective transformation of a clear star image. Depending on the motion parameters obtained by the navigation system, we can get the trajectory function of the blur kernel on image plane and then the blurred star image with rotations and angular vibrations can be obtained.

\section{Blurred star image restoration}

In this section, we describe how to use the Richardson-Lucy algorithm to restore the blurred star image. Richardson-Lucy algorithm, a nonlinear ratio-based method, is effective for processing Poisson noise in a star image and can avoid ringing artifacts that usually exist in linear restoration methods. It has the advantages of taking only a few minutes, even on large star images and always produces nonnegative gray-level values, which gives results that make better physical sense. This method maximizes a Poisson-statistics star image model likelihood function, given by[9]

$$
O^{t+1}(x)=O^{t}(x) \times K(-x) \otimes \frac{B(x)}{K(x) \otimes O^{t}(x)}
$$

where $B(x)$ is the measured blurred star image, $O^{t}(x)$ is the k-th estimation of the sharp image, $O^{0}(x)=B(x), K(x)$ is the blur kernel, $K(-x)$ is the transpose of that flips the shape of upside-down and left-to-right, $\otimes$ is the convolution operation.

Given the estimated blur kernel, the sharp star image can be obtained by Eq.(3). However, for a non-uniform blurred star image, the Richardson-Lucy algorithm is not suitable. In this case, we use the projective motion Richardson-Lucy algorithm [11] to restore the blurred star image. The general form of this algorithm is

$$
O^{t+1}(x)=O^{t}(x) \times \frac{1}{M} \sum_{i=1}^{M} E_{i}^{t}(-x)
$$

where $E_{i}^{t}(x)=\frac{B(x)}{\frac{1}{M} \sum_{i=1}^{M} O_{i}^{t}(x)}$ is the residual error between the real blurred image $\mathrm{B}$ and the

predicted blurred image $B^{t}=\frac{1}{M} \sum_{i=1}^{M} O_{i}^{t}(x)$. M is the number of accumulation. In deep-space 
exploration, SINS navigation system is introduced to improve the precision and stability of navigation system, so that certain motion information can be derived from SINS, including vehicle speed, angular rate etc. The trajectory can be obtained based on the information. Consequently, the restoration algorithm can be used to restore the blurred star image.

\section{Simulation and analysis}

Based on the simulation method, the blurred star image with rotation and angular vibration is shown in Fig.1

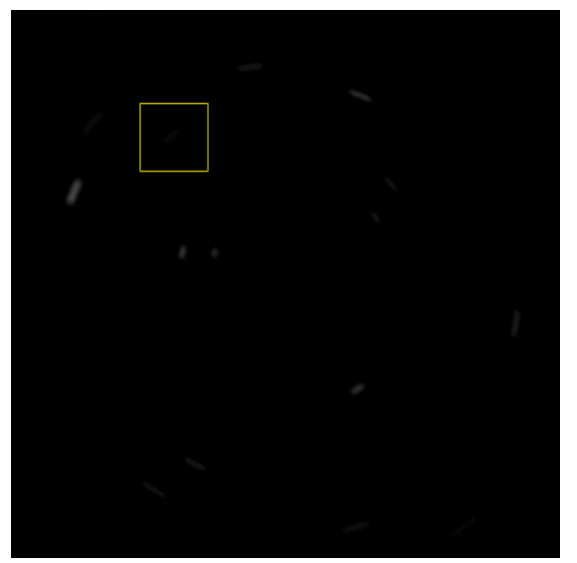

Fig. 1 The blurred star image with rotation and angular vibration.

where the displacement caused by rotation around $X_{C}$ axis is 10 pixels, the rotational angular velocity is $w_{z}=10^{\circ} / \mathrm{s}$ around $Z_{C}$ axis, the vibration amplitude is $A=3$ pixels, and its frequency is $f=100 \mathrm{~Hz}$ with an angle of $30^{\circ}$ to the horizontal, the exposure time is $400 \mathrm{~ms}$.

Fig. 2 shows the results of blurred star image and table1 shows the comparison of extraction errors before and after the restoration.

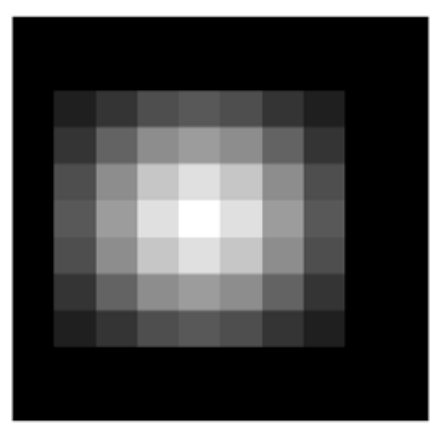

(a)

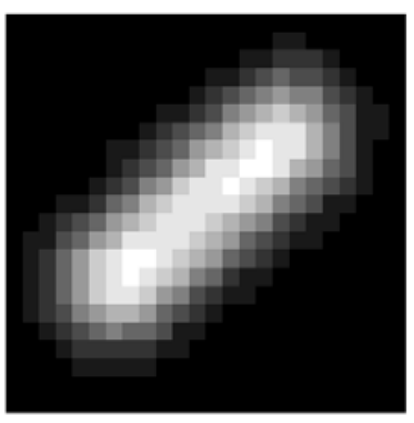

(b)

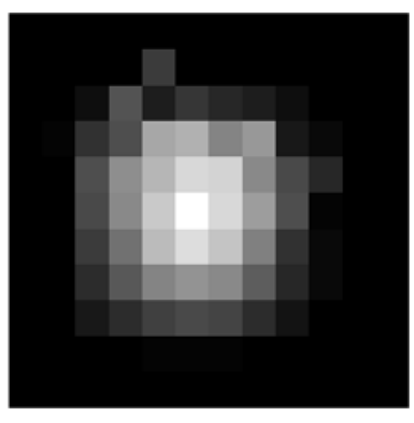

(c)

Fig.2 The comparison of shape and gray distribution of one star before and after restoration.(a) is the original star, (b)is the blurred star, (c) is the restored star. 
Table1 The comparison of the star centroid against blurred star image

\begin{tabular}{cccccc}
\hline $\begin{array}{c}\text { Index of } \\
\text { star spot }\end{array}$ & $\begin{array}{c}\text { Coordinate } \\
\text { of original } \\
\text { star image } \\
\text { (pixel) }\end{array}$ & $\begin{array}{c}\text { Coordinate of } \\
\text { blurred star } \\
\text { image } \\
\text { (pixel) }\end{array}$ & $\begin{array}{c}\text { Extraction } \\
\text { error } \\
\text { (pixel) }\end{array}$ & $\begin{array}{c}\text { Coordinate of } \\
\text { restored star } \\
\text { image } \\
\text { (pixel) }\end{array}$ & $\begin{array}{c}\text { Extraction } \\
\text { error } \\
\text { (pixel) }\end{array}$ \\
\hline 1 & $(181,55)$ & $(170.51,59.37)$ & $(-10.49,4.37)$ & $(180.71,55.20)$ & $(-0.29,0.20)$ \\
2 & $(115,69)$ & $(105.34,76.93)$ & $(-9.66,7.93)$ & $(115.07,69.13)$ & $(0.07,0.13)$ \\
3 & $(6,76)$ & $(4.08,80.48)$ & $(-1.92,4.48)$ & $(7.68,75.12)$ & $(1.68,-0.88)$ \\
4 & $(124,143)$ & $(118.25,150.25)$ & $(-5.75,7.25)$ & $(124.07,142.95)$ & $(0.07,-0.05)$ \\
5 & $(232,159)$ & $(226.72,160.55)$ & $(-5.28,1.55)$ & $(232.03,159.17)$ & $(0.03,0.17)$ \\
6 & $(231,189)$ & $(227.43,190.57)$ & $(-3.58,1.57)$ & $(231.02,189.02)$ & $(0.02,0.02)$ \\
7 & $(56,213)$ & $(54.06,223.85)$ & $(-1.94,10.85)$ & $(56.11,213.45)$ & $(0.11,0.45)$ \\
\hline
\end{tabular}

The local entropy method [12] is used to extract the centroid of star spots. It appears that the effects of rotation and angular vibration have great impact on the extraction accuracy of star spot and the extraction errors of $\Delta x$ or $\Delta y$ can be larger than ten pixels for star centroid without the restoration. After the restoration, the extraction errors of $\Delta x$ or $\Delta y$ are within subpixel range, which indicates that the effects caused by rotation and angular vibration can be ignored by the restoration.

\section{Conclusions}

The motion-blurred star image acquisition and restoration method are studied when the star tracker has long focal length and exposure time and small visual angle in this paper. Bade on simulation method, blurred star images are obtained and a restoration method is introduce to restore the blurred star image. After the restoration, the extraction error is within the subpixel level, indicating that the proposed method is both effective and feasible. The work in this paper provides the theory basis for improving the star tracker' dynamic performance and the celestial optical autonomous navigation accuracy.

\section{References}

[1] Liebe C C. Accuracy Performance of Star Trackers-A Tutorial, J. IEEE Transactions on Aerospace \& Electronic Systems. 38 (2002) 587-599.

[2] Jia H, Yang J K, Li X J, et al. Systematic error analysis and compensation for high accuracy star centroid estimation of star tracker, J. Science China Technological Sciences. 53 (2010) 3145-3152.

[3] Gong D, Yanpeng W U, Xin L U. An Attempt at Improving Dynamic Performance of Star Tracker by Motion Compensation, J. Aerospace Control \& Application, 2009.

[4] Fei X, Nan C, Zheng Y, et al. A Novel Approach Based on MEMS-Gyro's Data Deep Coupling for Determining the Centroid of Star Spot, J. Mathematical Problems in Engineering, 2012.

[5] Weina Z, Wei Q, Lei G. Blurred Star Image Processing for Star Sensors under Dynamic Conditions, J. Sensors. 12 (2012) 6712-26.

[6] Ting S, Fei X, Zheng Y, et al. Smearing model and restoration of star image under conditions of variable angular velocity and long exposure time, J. Optics Express. 22 (2014) 6009-6024.

[7] Wu X, Wang X. Multiple blur of star image and the restoration under dynamic conditions, J. Acta Astronautica. 68 (2011) 1903-1913.

[8] Wei Q, Weina Z. Restoration of Motion-blurred Star Image Based on Wiener Filter, C. Proceedings of the 2011 Fourth International Conference on Intelligent Computation Technology and Automation - Volume 02. IEEE Computer Society. 2011 691-694. 
[9] Chu Y L, Shao-Chun L I, Wang M. An Algorithm of Modified Richardson-Lucy Regularization Approach for Image Restoration, J. Journal of Langfang Teachers College, 2012.

[10] Wang Xiaoyan, Yan Jiqing, Tang Yi, et al. Simulation and analysis of high frequency vibration fuzzy image, J. optical technology. 37 (2011) 346-350.

[11] Tai Y W, Tan P, Brown M S. Richardson-Lucy Deblurring for Scenes Under Projective Motion Path, J. IEEE Transactions on Pattern Analysis \& Machine Intelligence. 33 (2010) 1603 1618.

[12] Tian Yulong, Wang Guangjun, Fang Jiancheng, et al. Star star star map extraction method based on local entropy, J. Journal of Harbin Institute of Technology. 37 (2005) 1068-1070. 